\title{
BMJ Open Study to Improve Cardiovascular Outcomes in high-risk older patieNts (ICON1) with acute coronary syndrome: study design and protocol of a prospective observational study
}

Vijay Kunadian, ${ }^{1,2}$ R Dermot G Neely, ${ }^{3}$ Hannah Sinclair, ${ }^{1,2}$ Jonathan A Batty, ${ }^{1,2}$ Murugapathy Veerasamy, ${ }^{1,2}$ Gary A Ford, ${ }^{4}$ Weiliang Qiu ${ }^{5}$

To cite: Kunadian V, Neely RDG, Sinclair $\mathrm{H}$, et al. Study to Improve Cardiovascular Outcomes in high-risk older patieNts (ICON1) with acute coronary syndrome: study design and protocol of a prospective observational study. BMJ Open 2016;6:e012091. doi:10.1136/bmjopen-2016012091

- Prepublication history and additional material is available. To view please visit the journal (http://dx.doi.org/ 10.1136/bmjopen-2016012091).

Received 29 March 2016 Revised 15 June 2016 Accepted 25 July 2016

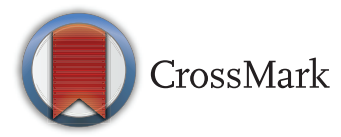

For numbered affiliations see end of article.

\section{Correspondence to} Dr Vijay Kunadian; vijay.kunadian@newcastle.ac. uk

\section{ABSTRACT}

Introduction: The ICON1 study (a study to Improve Cardiovascular Outcomes in high-risk older patieNts with acute coronary syndrome) is a prospective observational study of older patients ( $\geq 75$ years old) with non-ST-elevation acute coronary syndrome managed by contemporary treatment (pharmacological and invasive). The aim of the study was to determine the predictors of poor cardiovascular outcomes in this age group and to generate a risk prediction tool.

Methods and analysis: Participants are recruited from 2 tertiary hospitals in the UK. Baseline evaluation includes frailty, comorbidity, cognition and quality-oflife measures, inflammatory status assessed by a biomarker panel, including microRNAs, senescence assessed by telomere length and telomerase activity, cardiovascular status assessed by arterial stiffness, endothelial function, carotid intima media thickness and left ventricular systolic and diastolic function, and coronary plaque assessed by virtual histology intravascular ultrasound and optical coherence tomography. The patients are followed-up at 30 days and at 1 year for primary outcome measures of death, myocardial infarction, stroke, unplanned revascularisation, bleeding and rehospitalisation.

Ethics and dissemination: The study has been approved by the regional ethics committee (REC 12/NE/016). Findings of the study will be presented in scientific sessions and will be published in peerreviewed journals.

Trial registration number: NCT01933581: Preresults.

\section{INTRODUCTION}

In the general population, ischaemic heart disease (IHD) is the leading cause of death worldwide. ${ }^{1}$ Mortality due to IHD increases steeply among those aged $>70$ years. $^{2}$ In 2010, in the UK, more than twice as many individuals $>75$ years of age $(n=55028)$ died

\section{Strengths and limitations of this study}

- Older patients with non-ST-elevation acute coronary syndrome represent a high-risk population, who remain understudied in contemporary cardiovascular research.

- This prospective cohort study is designed and powered to identify risk factors for adverse outcomes, at 30 days and 1 year, in patients aged $\geq 75$ years undergoing invasive management of non-ST-elevation acute coronary syndrome.

- This study will evaluate the role of frailty, using a well-defined frailty index, and invasive imaging modalities (including optical coherence tomography and virtual histology intravascular ultrasound) as determinants of clinical outcome and also evaluate the quality of life in this age group.

- Limitations include (1) the non-randomised character of this study, which is not able to derive definitive insights regarding the causality of factors associated with clinical outcomes, and (2) that intracoronary imaging will be performed in only a subset of patients recruited, owing to anatomical contraindications and patient wishes.

- The results of this study will enable improved risk stratification for older patients presenting with non-ST-elevation acute coronary syndrome and will have implications for the design of future clinical trials in this high-risk population.

from IHD, compared to younger individuals $<75$ years $(\mathrm{n}=25540){ }^{3}$ According to the Myocardial Ischaemia National Audit Project Database annual public report 2012-2013, there were 80974 admissions with a final diagnosis of myocardial infarction (MI). Of these, $60 \%$ had non-ST-elevation myocardial infarction (NSTEMI). Of the patients with NSTEMI, $59 \%$ were $>70$ years of age $(26 \%$ were aged $70-79$ years, $26 \%$ were aged 80 89 years and $7 \%$ were aged $\geq 90$ years). ${ }^{4}$ 
Mortality benefit from advances in the management of acute coronary syndrome (ACS) has largely been realised in patients aged $<65$ years. ${ }^{2}$ There has been an increase in IHD burden in older patients, who are at risk of poorer outcomes due to frailty and comorbidity. ${ }^{5}$

Until recent years, there has persisted a paucity of evidence from clinical trials and studies to inform the management of ACS in older patients. More than half of all randomised controlled trials for ACS failed to enrol participants $>75$ years of age and, even in those that did, only $9 \%$ were $>75$ years of age. ${ }^{6}$ Notable studies, recruiting patients $>75$ years of age, have been reported in recent years, in the context of invasive and non-invasive management of ST-elevation MI and non-ST-elevation ACS. ${ }^{7-10}$ Evidence-based recommendations from trials do not account for age-related differences in physiology, disease and comorbidities, which may alter the risk-benefit profile of cardiovascular treatments and interventions. The age mismatch between trial and community populations begins at 75 years and widens with age. ${ }^{11}$ Furthermore, older people who are included in trials have lower than expected rates of traditional cardiovascular risk factors, fewer comorbidities and better renal function than the community population. ${ }^{12}$ Risks and benefits derived from trials cannot always be extrapolated to older patients in daily clinical practice due to the differences between the patient groups and their baseline characteristics. $^{13}$

In the ageing population, there is increasing evidence for the association of cardiovascular disease (CVD) and frailty. ${ }^{14}$ Depending on the frailty scale used and the population studied, almost half of the patients with CVD can be identified as frail. ${ }^{15}$ There is an increased risk of mortality and major adverse cardiovascular events in frail patients with CVD, especially those undergoing invasive procedures or suffering from coronary artery disease and heart failure. ${ }^{15}$ In patients aged $>75$ years, frailty was strongly and independently associated with in-hospital mortality (OR 4.6; 95\% CI 1.3 to 16.8 ) and 1 month mortality (OR 4.7; 95\% CI 1.7 to 13.0 ). ${ }^{16}$ At 1 year, there was a significant increase in mortality among frail patients compared with non-frail patients (HR 4.3, 95\% CI 2.4 to 7.8). ${ }^{17}$ Similarly, in $>65$-year-old patients, frailty was associated with increased long-term mortality and MI among patients undergoing percutaneous coronary intervention (PCI). ${ }^{18}$

No studies have been performed in older patients undergoing an invasive treatment strategy to evaluate predictors of poor outcomes or to develop strategies to improve outcomes following ACS. The ACS and PCI risk models that are currently available were mainly derived from patients $<65$ years of age and, hence, cannot be applied to the increasing proportion of older patients (aged $>75$ years) with ACS managed by contemporary treatment. $^{19}$ The goal of Improve Cardiovascular Outcomes in high-risk older patieNts with acute coronary syndrome (ICON1) study is to determine the predictors of adverse outcomes (death, MI, stroke, repeat, unplanned revascularisation, bleeding and rehospitalisation for any reason) at 1 month and at 1 year following invasive management of non-ST-elevation acute coronary syndrome (NSTEACS) in older patients and to develop an integrated risk score to predict adverse outcomes at 1 year that will inform clinical decision-making. In addition, the impact of contemporary NSTEACS management on the quality of life will be assessed.

\section{HYPOTHESIS}

Frailty and comorbid status in older patients are associated with worse outcomes following invasive treatment for NSTEACS.

\section{TRIAL DESIGN}

The study has been designed as a multicentre, prospective, observational study of patients aged $\geq 75$ years undergoing invasive management (coronary angiography with a view to revascularisation) for NSTEACS.

\section{METHODS}

\section{Study setting}

This ongoing, multicentre, observational study is being conducted in two tertiary cardiac care hospitals in the North-East of England. The Freeman Hospital, in Newcastle upon Tyne, is a tertiary cardiac centre with a catchment population of 2 million. Approximately 3000 PCI procedures are performed each year. The James Cook University Hospital, in Middlesbrough, performs $\sim 1750$ PCI procedures every year. The study participants are recruited from patients referred to these hospitals from the neighbouring district general hospitals for invasive treatment of NSTEACS. Patients are diagnosed on the basis of clinical symptoms, electrocardiography criteria and high-sensitivity troponin testing, in line with guidelines ${ }^{20} 21$ transferred the day before or on the day of procedure to the tertiary hospitals. Prospective ICON1 patients are identified from an electronic referral system and, on arrival to the tertiary hospitals, are approached for recruitment into the study. The research team explains the study to the patients and a patient information sheet is provided. If a patient agrees to participate in the study, a written informed consent is obtained. All patients screened for the study are entered in a screening $\log$, with details regarding the patients consented, declined and consented but not recruited (due to alternative diagnosis following coronary angiography). The inclusion and exclusion criteria are shown in box 1. Recruitment to the study started in October 2012 with the 1-year follow-up is projected to reach completion in December 2016.

\section{Treatment protocol}

During the course of the study, the patients were treated according to contemporary evidenced-based guidelines, as directed by an interventional cardiologist, at the time 


\section{Box 1 Inclusion and exclusion criteria}

Inclusion criteria:

- $\geq 75$ years old

- Non-ST-elevation acute coronary syndrome

- Planned for coronary angiogram (CA) or percutaneous coronary intervention

Exclusion criteria:

- Cardiogenic shock

- Primary arrhythmias

- Significant valvular heart disease

- Malignancy with life expectancy $<1$ year

Active infection:

- Urinary tract infection

- Pneumonia

- Sepsis

Alternative diagnosis after CA (excluded after consent):

- Pulmonary embolism

- Takotsubo cardiomyopathy

- Myocarditis

- Coronary vasospasm

Unable to consent:

- Known dementia

- Language barrier

- Visual impairment

- Lack of capacity

of study enrolment. ${ }^{20} 21$ According to standard practice, the patients are revascularised by PCI or coronary artery bypass graft surgery. The patients may also be managed medically, if deemed not appropriate for either of the revascularisation strategies at the discretion of the operating cardiologist.

\section{Data collection}

Data are collected on standardised case report forms by members of the research team. The data collected include demographics, baseline characteristics, and details of coronary angiography and/or PCI. Periprocedural complications and in-hospital complications are recorded. Further data are collected on the cardiovascular status, Canadian Cardiovascular Society (CCS) angina grade, New York Heart Association (NYHA) dyspnoea grade, frailty category, functional health status, quality of life and cognitive status. These are listed in box 2. The assessments and techniques used for the above data collection are discussed in the following sections. The study flow chart is displayed in figure 1. All questionnaires were administered verbally, in person and by a trained, clinical researcher. Appropriate training was provided to researchers, ensuring that these scripted questionnaires were performed, and results recorded, in an unbiased fashion.

\section{Frailty and comorbidity assessments}

Frailty is assessed by Fried Frailty Index, derived from Cardiovascular Health Study ${ }^{22}$ and Rockwood Frailty Index, derived from Canadian Study of Health and

\section{Box 2 ICON1 study assessments}

Biomarkers:

- High-sensitive $C$ reactive protein

- Vitamin D

- Myeloperoxidase

- Asymmetric dimethyl arginine

- Eicosapentaenoic acid

- Docosahexaenoic acid

- Soluble p selectin

- Cluster differentiation 40

- Lipoprotein-associated phospholipase $\mathrm{A}_{2}$

- Interleukin-6

- Tumour necrosis factor- $\alpha$

- N-terminal prohormone brain natriuretic peptide

- MicroRNAs (miR-21-5p, miR-126-5p, miR-132-3p, miR-133a-3p, miR-142-3p, miR-150-5p, miR-208-3p, miR-223-3p and miR-320a)

- Peripheral blood mononuclear cells - Telomere length

- Telomerase activity

Intracoronary imaging:

- Virtual histology intravascular ultrasound

- Optical coherence tomography

Cardiovascular status:

- Arterial stiffness

- Peripheral arterial tonometry

- Carotid intima media thickness

- Transthoracic echocardiogram

Cardiac symptoms:

- New York Heart Association dyspnoea

- Canadian Cardiovascular Society angina

Frailty assessment:

- Fried Frailty Index

- Rockwood Frailty Index

Quality of life (Qol):

- SF-36, EuroQol-5D (EQ-5D)

Cognitive status:

- Montreal Cognitive Assessment (permission to use MoCA test obtained from MoCA team (on behalf of $\mathrm{Dr}$ Ziad Nasreddine))

Comorbidity:

- Charlson Comorbidity Index

Aging. ${ }^{23}$ The Fried Frailty Index is based on assessing five criteria, comprising subjective answers provided by the patient (regarding weight loss, physical energy, physical activity) and objective assessment (hand grip strength). A score of 0 is categorised as robust, 1 or 2 as intermediate or pre-frail and 3 or more as frail (see online supplementary appendix 1). The Rockwood Frailty Index is, based on the assessment by the researchers, grouped into categories $1-7$, from very fit to severely frail, depending on functional status and independence/dependence on others for activities of daily living (see online supplementary appendix 2).

In addition, the Charlson Comorbidity Index, ${ }^{24}$ a method of predicting mortality based on a weighted index of the number and seriousness of comorbid conditions, is evaluated for each patient. The Charlson 
Figure 1 ICON1 study flow chart. CCS, Canadian

Cardiovascular Society; ICON1, Improve Cardiovascular

Outcomes in high-risk older

patieNts with acute coronary

syndrome; MoCA, Montreal

Cognitive Assessment; NSTEMI,

non-ST-elevation acute coronary

syndrome; NYHA, New York

Heart Association; OCT, optical

coherence tomography; $\mathrm{PCl}$,

percutaneous coronary

intervention; UA, unstable angina;

VH-IVUS, virtual histology-

intravascular ultrasound.

\section{ICON 1: Consenting $75^{+}$patients admitted with UA/NSTEMI}

Coronary Angiography and decision to $\mathrm{PCl}$

Blood samples for biomarker analysis

$\&$

VHIVUS culprit vessel pre and post $\mathrm{PCl}+$

$\mathrm{VH}$ IVUS+OCT in all 3 coronaries where feasible

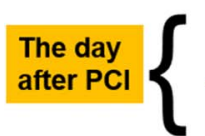

Charlson co-morbidity index, Frailty Scores, QOL measures, NYHA, CCS class, MOCA

Ankle Brachial Index, Vascular Stiffness, Carotid Intima Media Thickness, Peripheral Arterial Tonometry, Echo

Primary Endpoint: Death, Myocardial Infarction, Stroke, Repeat

Revascularisation, Bleeding, Rehospitalisation for any reason
Comorbidity Index has been demonstrated to be an appropriate indicator of in-hospital and 1-year outcomes in the setting of ACS. ${ }^{25}$

\section{Functional status and quality-of-life measures}

The Short Form-36 Standard (SF-36 Standard) health survey is completed by each patient prior to discharge from the hospital and at 1-year follow-up to assess functional health and quality of life. The responses will be used to obtain physical component summary score and mental component summary score. ${ }^{26}$ In addition, the EQ-5D-3L questionnaire is used to assess the health outcome of each patient at discharge and 1-year follow-up. ${ }^{27} 28$

\section{Cognitive status assessment}

Atherosclerosis is associated with increased risk of cognitive impairment in older patients. ${ }^{29}$ To assess the cognitive status of patients during admission, the Montreal Cognitive Assessment (MoCA) ${ }^{30}$ test is used. The MoCA has been shown to have high sensitivity in screening patients with known CVD for mild cognitive impairment, even in a non-memory clinic setting. ${ }^{31}$ This test is repeated at 1-year follow-up.

\section{Biomarker sampling}

Blood samples are collected at the time of coronary angiography and/or PCI for biomarker analysis. Serum for biomarkers is stored for analysis in batches. Peripheral blood mononuclear cells are separated by centrifugation techniques for storage at $-80^{\circ} \mathrm{C}$ for analysis of telomeres and telomerase activity. High-sensitivity $\mathrm{C}$ reactive protein (hsCRP), parathyroid hormone and total vitamin D are analysed. Full blood count, renal function, blood glucose, cholesterol and high-sensitivity cardiac troponin $\mathrm{T}$ levels are measured in patients as part of our routine care.

Inflammation plays a central role in acute thrombotic complications of unstable atherosclerotic coronary plaque. Increased levels of markers of inflammation predict CV outcomes following ACS. Inflammatory markers including myeloperoxidase, ${ }^{32} \mathrm{hsCRP}^{33}$ and soluble CD40 ligand ${ }^{34}$ have been associated with ACS and have been shown to predict the outcome. The patients with ACS have decreased levels of anti-inflammatory $\omega-3$ fatty acids (eicosapentaenoic acid and docosahexaenoic acid). ${ }^{35}$ Increased lipoprotein-associated phospholipase $\mathrm{A}_{2}$ activity has been associated with increased cardiovascular event rates. ${ }^{36}{ }^{37}$ An elevated level of asymmetric dimethyl arginine is a strong and independent predictor of adverse outcomes following ACS. ${ }^{38}$ Interleukin-6 (IL-6) levels in the serum were increased in patients with ACS. ${ }^{39}$ IL-6 expressed in atherosclerotic plaques may increase plaque instability. ${ }^{40}$ Elevated IL-6 was a predictor of 6-month and 12-month mortality in patients with unstable coronary artery disease. ${ }^{41}$ Tumour necrosis factor- $\alpha(\mathrm{TNF}-\alpha)$ is a proinflammatory cytokine associated with myocardial dysfunction and remodelling following ACS. ${ }^{42}$ In patients with recent MI, increased levels of TNF- $\alpha$ were associated with adverse cardiovascular outcomes (recurrent MI and cardiac death). ${ }^{43}$ Vitamin D deficiency has been associated with elevated CAD burden and worse cardiovascular outcomes. ${ }^{44}$ These biomarkers will be analysed in this group of $\geq 75$-year-old patients to enable the determination of predictors of adverse CV outcomes at 1 year. Telomere shortening has been associated with ageing and senescence, and shorter leucocyte telomeres are associated with increased cardiovascular risk and mortality. ${ }^{45}$ Shorter leucocyte telomere length predicted high-risk plaque morphology on virtual histology intravascular ultrasound (VH-IVUS). ${ }^{46}$ Whether shorter telomere length is a predictor of adverse events among older patients undergoing PCI is not known and will be evaluated in this study.

\section{MicroRNA analysis}

MicroRNAs (miRNAs) are small non-coding RNAs that post-transcriptionally inhibit gene expression. ${ }^{47}$ In the 
past few years, miRNAs have emerged as key tools for the understanding of IHD pathophysiology, with great potential to be used as new biomarkers and therapeutic targets. miRNAs seem to possess ideal characteristics to be used as disease biomarkers, as they are detectable in biofluids in a reproducible and stable fashion, even after years of sample storage and freeze-thaw cycles. ${ }^{48}$ In the blood, circulating miRNAs are found mainly within extracellular vesicles, such as exosomes, microvesicles and apoptotic bodies, ${ }^{49}$ and, to a lesser extent, associated with HDL-cholesterol particles ${ }^{50} 51$ or Argonaute-2 protein. ${ }^{52}$ Several studies have demonstrated elevated or decreased levels of specific circulating miRNAs in patients with ACS. ${ }^{53-56}$ However, few have addressed their prognostic value with regards to major cardiovascular events ${ }^{57}$ or death ${ }^{58}$ especially among older cohorts of patients presenting with NSTEACS.

The levels of nine circulating miRNAs, known to be differentially expressed in patients with ACS (miR-21-5p, miR-126-5p, miR-132-3p, miR-133a-3p, miR-142-3p, miR-150-5p, miR-208-3p, miR-223-3p and miR-320a), will be quantified by reverse transcription quantitative PCR, in serum and circulating microvesicles (isolated from an additional $200 \mu \mathrm{L}$ of serum) and correlated with clinical variables with a view to assess their value as a prognostic biomarker in older patients with NSTEACS.

\section{Invasive coronary artery imaging}

Postmortem studies have identified that vulnerable plaques, with specific morphological characteristics, are implicated in the pathophysiology of ACS. These plaques, which are prone to erosion and rupture, have inflamed fibrous caps, rich in macrophages, overlying a lipid pool. ${ }^{59}$ Burke et al ${ }^{60}$ examined the hearts of 113 men who had died suddenly and found that $95 \%$ of ruptured plaques had fibrous caps $<65 \mu \mathrm{m}$ thick (mean thickness $23 \pm 19 \mu \mathrm{m}$ ) with an infiltrate of macrophages. ICON1 aims to identify whether the increased mortality in the older population with ACS is due to an increased prevalence of these vulnerable thin-capped fibroatheroma (TCFAs). Following diagnostic coronary angiography, the patients undergo VH-IVUS imaging and optical coherence tomography (OCT) imaging in all three coronary arteries prior to PCI, where feasible and not contraindicated, and VH-IVUS imaging post-PCI in the culprit vessel at the discretion of the operating cardiologist.

\section{Virtual histology intravascular ultrasound}

The greyscale IVUS image uses only the amplitude of the reflected ultrasound wave. VH-IVUS uses spectral analysis of the frequency and power of the reflected wave to generate a more accurate reflection of the tissue subtypes present within the vessel wall. ${ }^{61}$ This can then be used to differentiate plaque components (fibrous, fibro-fatty, dense calcium and necrotic core) and identify high-risk vulnerable plaques. Although VH-IVUS lacks the resolution to identify the thin fibrous cap of the
TCFA, it is well placed to accurately identify the necrotic core of these plaques. ${ }^{61}$ A $20 \mathrm{MHz}$, phased-array Eagle Eye Platinum catheter is mounted on an R-100 pullback device and connected to either an integrated S5i system or a mobile S5 tower. Image acquisition is performed at a pullback speed of $0.5 \mathrm{~mm} / \mathrm{s}$ and is ECG-gated to ensure one frame is acquired per cardiac cycle. The maximum length of all three coronary arteries is imaged, where feasible and not contraindicated. ${ }^{62}$ The data are anonymised and transferred to DVD for off-line data analysis. The operator is blinded to these data.

VH-IVUS data analysis is performed using the Medis QIvus software v2.0 (Leiden, the Netherlands). Contours are drawn manually around the external elastic membrane and lumen of the vessel for each greyscale IVUS frame, excluding any ring-down artefact or previously stented segments. The software then calculates several parameters such as minimum lumen area and diameter, per cent stenosis, and absolute volume and percentage of each plaque component. The image reader can also calculate the remodelling index ${ }^{63}$ and classify the lesion type from these data. Lesion classification in ICON1 is based on previously published recommendations for tissue characterisation by radiofrequency data analysis (figure 2). ${ }^{62}$

\section{Optical coherence tomography}

OCT generates an image analogous to IVUS using a low coherence, near-infrared (wavelength $1.3 \mu \mathrm{m}$ ) light source, instead of sound. ${ }^{64}$ A bloodless field inside the coronary artery is vital, as red blood cells strongly backscatter the near-infrared light. This is obtained by using a flush of contrast during image acquisition. OCT has a greater resolution than IVUS (20-40 vs $100-200 \mu \mathrm{m})$ and is thus able to delineate the thin fibrous cap present in a TCFA. However, its poorer penetration (1-2.5 mm) can limit its capacity to identify deep lipid pools and quantify plaque volume. ${ }^{65} 66$

OCT images are obtained using a Dragonfly catheter (St Jude Medical, Minnesota, USA) connected to the Ilumien PCI Optimization System. Just before image acquisition, a short flush of iso-osmolar contrast is administered to ensure that the guide catheter is well engaged with the coronary artery and the catheter is clear of blood. The system is calibrated and OCT pullback is initiated with a further flush of iso-osmolar contrast $(10 \mathrm{~mL}$ in the right coronary artery and $15 \mathrm{~mL}$ in the left coronary artery). OCT images are obtained in $54 \mathrm{~mm}$ segments at a pullback rate of $20 \mathrm{~mm} / \mathrm{s}$ in all three coronary arteries, where feasible. Data are transferred anonymously to a DVD for off-line analysis; the operator is blinded to these data during the procedure.

OCT data are analysed using the Medis QIvus software. Contours are drawn around the lumen to generate data on the minimum lumen area and diameter. The whole vessel is then analysed to identify plaque subtypes. An atherosclerotic lesion is seen on OCT as a mass lesion within the arterial wall, with focal intimal thickening or loss of the normal vessel architecture. ${ }^{67}$ Fibrous plaque is 


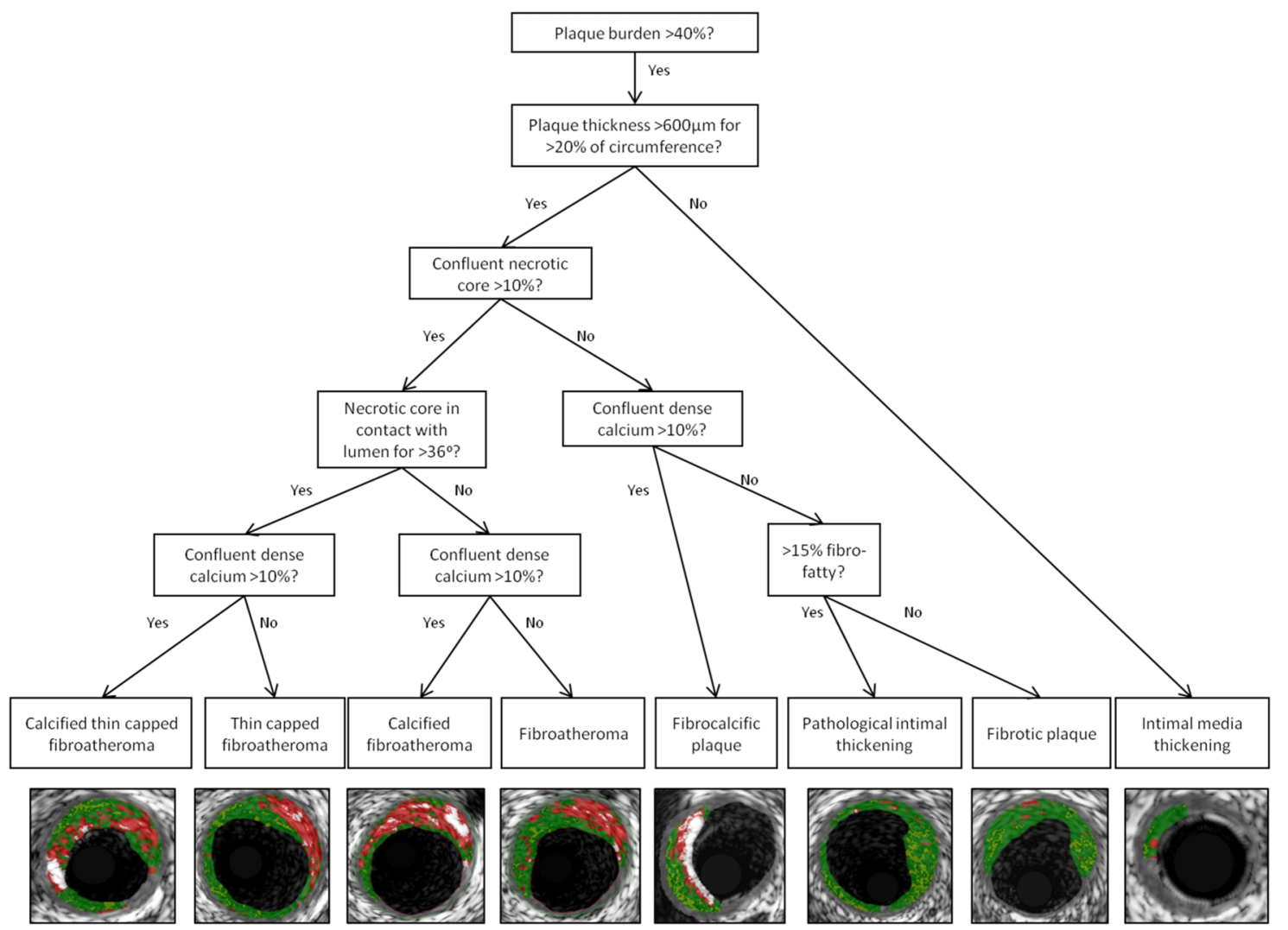

Figure 2 Decision tree for lesion classification on VH-IVUS, virtual histology-intravascular ultrasound (VH-IVUS) with image examples. Adapted from García-García et al. ${ }^{62}$

homogenous and highly backscattering, calcified plaques are signal-poor areas with sharply delineated borders and lipid pools are signal-poor regions with poorly defined borders and a fast OCT signal drop-off. ${ }^{67}$ Using side branches and areas of calcification as landmarks, it is possible to compare the accuracy of lesion subtypes identified by VH-IVUS and OCT.

\section{NON-INVASIVE ASSESSIMENT OF CARDIOVASCULAR STATUS \\ Arterial stiffness}

Arterial stiffness is now increasingly recognised as a surrogate end point for the assessment of CVD status. ${ }^{68}$ It can lead to angina in the presence of even minor coronary artery disease and to the development of diastolic dysfunction, the commonest form of heart failure in the elderly. ${ }^{69}$ Arterial stiffness is determined by carotid-femoral pulsewave velocity (PWV), which is a simple, non-invasive, robust and reproducible investigation method that can be performed at the bedside. ${ }^{68}$ In older patients, arterial stiffness assessed by increased PWV is associated with poor cardiovascular outcomes. ${ }^{70}$ In the ICON1 study, carotid-femoral PWV is assessed by the Vicorder device (Skidmore Medical Limited, Bristol, UK). In addition, brachiofemoral PWV, pulse-wave analysis (includes pulse pressure, augmentation pressure and augmentation index) and ankle brachial pressure index are also assessed.

\section{Endothelial function}

Endothelial dysfunction is considered one of the earliest markers of atherosclerosis, ${ }^{71}$ contributing to lesion development and its later clinical manifestations. ${ }^{72}{ }^{73}$ It is associated with increased risk of cardiovascular events and has been proposed as a marker of poor $\mathrm{CV}$ outcomes. ${ }^{74-76}$ Peripheral arterial tonometry (PAT) by finger plethysmography (EndoPAT; Itamar Medical, Caesarea, Israel) is a novel method of measuring the peripheral vasodilator response. ${ }^{77}$ Hyperaemic response measured by PAT signal amplitude gives a measure of nitric oxide-mediated endothelial function. ${ }^{79} 80$ In patients with low-risk findings during stress testing and/or the absence of new obstructive lesions on angiography, lower natural logarithmic-scaled reactive hyperaemia index $(<0.40)$ is associated with increased cardiovascular death over 6 years. ${ }^{81}$ In the ICON1 study, endothelial function is measured by EndoPAT. PAT signals are recorded from the index fingers with pneumatic probes at baseline, during cuff occlusion and during hyperaemia. A measure of endothelial function is calculated from the ratio of PAT signal amplitude at baseline and postocclusion. Reactive hyperaemia index data from the study will be used in the prediction of adverse CV outcomes and will be incorporated in the risk model.

\section{Carotid intima media thickness}

Carotid intima media thickness (CIMT) is a significant predictor of incident adverse cardiovascular events. ${ }^{82} 83$ 
Increased CIMT was associated with severity of coronary atherosclerosis in ACS. ${ }^{84}$ CIMT and its association with predicting CV events in older patients with NSTEACS are not known. In a meta-analysis, addition of CIMT to Framingham risk score in general population did not improve 10-year prediction of first $\mathrm{MI}$ or stroke. ${ }^{85}$ However, CIMT and arterial stiffness together increase the cardiovascular risk in patients with known vascular disease or cardiovascular risk factors. ${ }^{86}$ In the ICON1 study, CIMT is assessed using a Vivid I GE machine, with a vascular probe. CIMT measurement is obtained via semiautomated software, which uses an edge detection technique. CIMT values will be analysed for the prediction of adverse outcomes and will be incorporated in the risk model.

\section{Transthoracic echocardiogram}

In hospitalised elderly patients with known CVD, left ventricular diastolic dysfunction was similar in prevalence to systolic dysfunction and was associated with similar cardiovascular and all-cause mortality. ${ }^{87}$ Transthoracic echocardiography will be performed using a Vivid I GE echo machine, according to the British Society of Echocardiography guidelines, to assess systolic function, diastolic function and valvular heart disease. ${ }^{88}$ Systolic and diastolic function will be analysed for the prediction of adverse CV outcomes.

\section{Follow-up}

One-month outcomes are recorded using general practitioner summary documents, obtained from the patients' general practitioner. The patients are followed-up in a study outpatient clinic at 1 year. During this follow-up visit, repeat blood samples are collected for biomarker analysis. In addition, NYHA class, CCS angina class, SF-36, EQ-5D and MoCA assessments are completed. Frailty status is reassessed using Fried and Rockwood Frailty Criteria.

\section{Primary outcome measures}

The primary outcome measure is a composite of death, MI, stroke, repeat, unplanned revascularisation and BARC (Bleeding Academic Research Consortium)-defined bleeding (type 2 or greater) at 1 year (see online supplementary appendices 3 and 4). ${ }^{89}$ We also intend to analyse 1-year mortality as an independent outcome measure. All-cause hospitalisation comprises a secondary outcome measure.

\section{Sample size}

For the primary outcome, Hsieh and Lavori's ${ }^{91}$ method was used to calculate the power for testing the association of the risk score with adverse outcomes, based on 300 participants with a type I error rate of 0.05 . From the nationallevel registry data, the 1-year mortality rate for NSTEMI in all patients undergoing invasive strategy is $\sim 2-5 \% .^{92}$ Estimates of the SD and HR of the risk score are unknown. An assumption was made on the HRs being an increment of $1 \mathrm{SD}$ of the risk score (see figure 3 ).

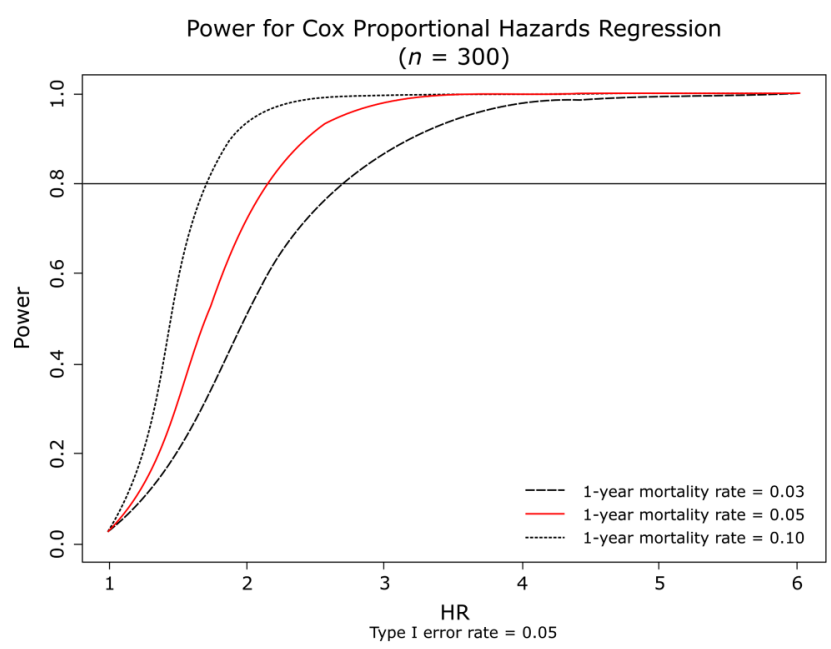

Figure 3 Study power. A plot of power versus hazard ratios for the sample size of 300 patients and 1-year mortality rates.

\section{STATISTICAL METHODS}

\section{Risk factor selection}

Cox proportional hazards regression analysis will be performed to estimate HRs of the risk factors and associated $p$ values for the primary outcome. Multiple logistic regression analysis will be performed to estimate ORs of the risk factors and associated $\mathrm{p}$ values for the secondary outcome. The bootstrap method will be used to avoid overfitting the data. One thousand bootstrapping will be performed. For each bootstrapping, we will sample with replacement 300 patients from the original 300 patients. Backward selection with a $p$ value of $<0.05$ for statistical significance will be used to remove variables in each sample. Variables selected $\geq 800$ times $(80 \%)$ in the overall sample will be included in the final model. All missing values will be reported, and appropriate statistical methods will be used to handle missing values.

\section{Risk score construction}

To construct the risk score, risk factors identified through the multivariable model will be assigned a weight. Weights are the estimated regression coefficients from the Cox proportional hazards regression or logistic regression model. The risk score is thus the weighted average of the identified risk factors. Another Cox proportional hazards regression or logistic regression model will be applied to detect the association of the proposed risk score to the outcomes.

\section{Risk score evaluation}

Harrell's C-index will be used to assess the discriminatory capacity of the integrated risk score for primary and secondary outcomes. The Jackknife method will be used to estimate the SE of the estimated Harrell's C-index ${ }^{93}$ or area under the curve. The difference between modelpredicted and observed event rates (goodness-of-fit) will be evaluated using the Hosmer-Lemeshow test ( $p$ value of $>0.10$ will be considered to indicate the lack of 
deviation between the model-predicted and observed event rates). Reclassification calibration measures (eg, net reclassification improvement and integrated discrimination improvement) will be used to evaluate the improvement of new predictors (relative to existing predictors) on the agreement between observed and predicted outcomes. ${ }^{94}$ A cross-validation technique will be used to assess how the results of statistical analysis generalise to an independent data set. ${ }^{95}$ Finally, a prediction nomogram ${ }^{96}$ will be developed to facilitate calculating the risk scores and the corresponding survival probability at 1 year.

\section{CONCLUSION}

The ICON1 study will identify predictors of poor cardiovascular outcomes among older patients (aged $\geq 75$ years) presenting with NSTEACS managed by contemporary pharmacotherapy and invasive revascularisation strategy. Based on clinical characteristics, frailty status, comorbidities and cardiovascular status, an integrated risk stratification tool to help decision-making in the management of older patients will be developed. The variables that we hypothesise may be relevant to such a model would be either (1) routinely collected in clinical practice as part of current evidence-based practice or (2) should not be unduly burdensome to collect during routine clinical assessment.

\section{Author affiliations}

${ }^{1}$ Institute of Cellular Medicine, Newcastle University, Newcastle upon Tyne, UK ${ }^{2}$ Cardiothoracic Centre, Freeman Hospital, Newcastle upon Tyne, UK

${ }^{3}$ Department of Biochemistry, Newcastle upon Tyne Hospitals NHS Foundation Trust, Newcastle upon Tyne, UK

${ }^{4}$ Institute for Ageing and Health, Newcastle University, Newcastle upon Tyne, UK

${ }^{5}$ Channing Division of Network Medicine, Brigham and Women's Hospital and Harvard Medical School, Boston, Massachusetts, USA

Twitter Follow Jonathan Batty at @jonnybatty

Acknowledgements The authors thank Dr J Ahmed, Dr A Bagnall, Dr R Das, Dr R Edwards, Dr M Egred, Dr I Purcell, Professor I Spyridopoulos and Professor A Zaman of Freeman Hospital, Newcastle upon Tyne, and Dr Mark de Belder and Mrs Bev Atkinson of the James Cook University Hospital, South Tees Hospitals NHS Foundation Trust, Middlesbrough, UK for their help with data collection. They also thank the Cardiology CRN research team at Freeman Hospital, Mrs Kathryn Proctor and Mrs Jennifer Adams-Hall, for their support with the follow-up of study patients. Dr Carmen Martin-Ruiz and Dr Gabriele Saretzki of Institute for Ageing and Health, Newcastle University, Newcastle upon Tyne, UK are thanked for their support with the telomere studies. The authors also acknowledge the Newcastle angiographic core laboratory members V K, H S, Kimberley Batanghari, Dhiluni Kandage, Jin Tee, Ross Fowkes, Victor Tsoi, Benjamin Beska and Rebecca Jordan and the Newcastle intravascular ultrasound/OCT core laboratory members V K, H S, Shristy Subba and James Latimer ; and Professor Gary Mintz, Cardiovascular Research Foundation, New York for their expert oversight of the invasive substudy.

Contributors VK conceived the study and carries the overall responsibility for the full study and the study protocol. DN is responsible for the biomarker substudy. HS contributed to the invasive substudy. JAB is responsible for overall critical review and revision of the manuscript. MV contributed to the non-invasive substudy and the initial draft of this manuscript. GAF provided expert input into the design of the protocol and critical review of the manuscript. WQ responsible for the statistical aspect of the study and the design of the study.

Funding The research is supported by the National Institute for Health Research (NIHR) Newcastle Biomedical Research Centre based at Newcastle-upon-Tyne Hospitals NHS Foundation Trust and Newcastle University. This study is also supported by unrestricted research support from the Volcano Corporation, San Diego, USA and Itamar Medical, Caesarea, Israel and St.Jude Medical UK.

Disclaimer The views expressed are those of the authors and not necessarily those of the NHS, the NIHR or the Department of Health.

Competing interests None declared.

Patient consent Obtained.

Ethics approval The study has been approved by the regional ethics committee (REC 12/NE/016) and is conducted in accordance with the Declaration of Helsinki (64th World Medical Association General Assembly, Fortaleza, Brazil, October 2013). ${ }^{97}$

Provenance and peer review Not commissioned; externally peer reviewed.

Open Access This is an Open Access article distributed in accordance with the Creative Commons Attribution Non Commercial (CC BY-NC 4.0) license, which permits others to distribute, remix, adapt, build upon this work noncommercially, and license their derivative works on different terms, provided the original work is properly cited and the use is non-commercial. See: http:// creativecommons.org/licenses/by-nc/4.0/

\section{REFERENCES}

1. Murray CJ, Lopez AD. Mortality by cause for eight regions of the world: Global Burden of Disease Study. Lancet 1997;349:1269-76.

2. Finegold JA, Asaria P, Francis DP. Mortality from ischaemic heart disease by country, region, and age: statistics from World Health Organisation and United Nations. Int J Cardiol 2013;168:934-45.

3. Townsend N, Wickramasinghe K, Bhatnagar P, et al. Coronary heart disease statistics 2012 edition. London: British Heart Foundation, 2012.

4. Gavalova L, Weston C. Myocardial Ischaemia National Audit Project Annual Public Report April 2012-March 2013. London: National Institute for Cardiovascular Outcomes Research, 2013.

5. Veerasamy M, Edwards R, Ford G, et al. Acute coronary syndrome among older patients: a review. Cardiol Rev 2015;23:26-32.

6. Lee PY, Alexander KP, Hammill BG, et al. Representation of elderly persons and women in published randomized trials of acute coronary syndromes. JAMA 2001;286:708-13.

7. Bueno $\mathrm{H}$, Betriu $\mathrm{A}$, Heras $\mathrm{M}$, et al, TRIANA Investigators. Primary angioplasty vs. fibrinolysis in very old patients with acute myocardial infarction: TRIANA (TRatamiento del Infarto Agudo de miocardio eN Ancianos) randomized trial and pooled analysis with previous studies. Eur Heart J 2011;32:51-60.

8. Savonitto S, Cavallini C, Petronio AS, et al. Early aggressive versus initially conservative treatment in elderly patients with non-ST-segment elevation acute coronary syndrome: a randomized controlled trial. JACC Cardiovasc Interv 2012:5:906-16.

9. Tegn N, Abdelnoor M, Aaberge L, et al, After Eighty study investigators. Invasive versus conservative strategy in patients aged 80 years or older with non-ST-elevation myocardial infarction or unstable angina pectoris (After Eighty study): an open-label randomised controlled trial. Lancet 2016;387:1057-65.

10. Roe MT, Goodman SG, Ohman EM, et al. Elderly patients with acute coronary syndromes managed without revascularization: insights into the safety of long-term dual antiplatelet therapy with reduced-dose prasugrel versus standard-dose clopidogrel. Circulation 2013;128:823-33.

11. Alexander KP, Newby LK, Cannon CP, et al. Acute coronary care in the elderly, part I: non-ST-segment-elevation acute coronary syndromes: a scientific statement for healthcare professionals from the American Heart Association Council on Clinical Cardiology: in collaboration with the Society of Geriatric Cardiology. Circulation 2007;115:2549-69.

12. Kandzari DE, Roe MT, Chen AY, et al. Influence of clinical trial enrollment on the quality of care and outcomes for patients with non-ST-segment elevation acute coronary syndromes. Am Heart $J$ 2005;149:474-81. 
13. Tinetti ME, Bogardus ST Jr, Agostini JV. Potential pitfalls of disease-specific guidelines for patients with multiple conditions. N Engl J Med 2004;351:2870-4.

14. Afilalo J, Karunananthan S, Eisenberg MJ, et al. Role of frailty in patients with cardiovascular disease. Am J Cardiol 2009;103:1616-21.

15. Afilalo J. Frailty in patients with cardiovascular disease: why, when, and how to measure. Curr Cardiovasc Risk Rep 2011;5:467-72.

16. Ekerstad N, Swahn E, Janzon M, et al. Frailty is independently associated with short-term outcomes for elderly patients with non-ST-segment elevation myocardial infarction. Circulation 2011;124:2397-404.

17. Ekerstad N, Swahn E, Janzon M, et al. Frailty is independently associated with 1-year mortality for elderly patients with non-ST-segment elevation myocardial infarction. Eur J Prev Cardiol 2014;21:1216-24

18. Singh M, Rihal CS, Lennon RJ, et al. Influence of frailty and health status on outcomes in patients with coronary disease undergoing percutaneous revascularization. Circ Cardiovasc Qual Outcomes 2011;4:496-502.

19. Bawamia B, Mehran R, Qiu W, et al. Risk scores in acute coronary syndrome and percutaneous coronary intervention: a review. $\mathrm{Am}$ Heart J 2013;165:441-50.

20. Hamm CW, Bassand JP, Agewall S, et al. ESC Guidelines for the management of acute coronary syndromes in patients presenting without persistent ST-segment elevation: The Task Force for the management of acute coronary syndromes (ACS) in patients presenting without persistent ST-segment elevation of the European Society of Cardiology (ESC). Eur Heart $J$ 2011;32:2999-3054.

21. Roffi M, Patrono C, Collet JP, et al. 2015 ESC Guidelines for the management of acute coronary syndromes in patients presenting without persistent ST-segment elevation: Task Force for the Management of Acute Coronary Syndromes in patients presenting without persistent ST-segment elevation of the European Society of Cardiology (ESC). Eur Heart J 2016;37:267-315.

22. Fried LP, Tangen CM, Walston J, et al. Frailty in older adults: evidence for a phenotype. J Gerontol A Biol Sci Med Sci 2001;56: M146-56.

23. Rockwood K, Song X, MacKnight C, et al. A global clinical measure of fitness and frailty in elderly people. CMAJ 2005;173:489-95.

24. Charlson ME, Pompei P, Ales KL, et al. A new method of classifying prognostic comorbidity in longitudinal studies: development and validation. J Chronic Dis 1987;40:373-83.

25. Radovanovic D, Seifert B, Urban P, et al. AMIS Plus Investigators. Validity of Charlson Comorbidity Index in patients hospitalised with acute coronary syndrome. Insights from the nationwide AMIS Plus registry 2002-2012. Heart 2014;100:288-94.

26. Ware JE Jr, Sherbourne CD. The MOS 36-item short-form health survey (SF-36). I. Conceptual framework and item selection. Med Care 1992;30:473-83.

27. EuroQOL Group. EuroQol-a new facility for the measurement of health-related quality of life. Health Pol 1990;16:199-208.

28. Brooks R. EuroQol: the current state of play. Health Pol 1996;37:53-72.

29. van Oijen M, de Jong FJ, Witteman JC, et al. Atherosclerosis and risk for dementia. Ann Neurol 2007;61:403-10.

30. Nasreddine ZS, Phillips NA, Bédirian V, et al. The Montreal Cognitive Assessment, MoCA: a brief screening tool for mild cognitive impairment. J Am Geriatr Soc 2005;53:695-9.

31. McLennan SN, Mathias JL, Brennan LC, et al. Validity of the Montreal cognitive assessment (MoCA) as a screening test for mild cognitive impairment $(\mathrm{MCl})$ in a cardiovascular population. $J$ Geriatr Psychiatry Neurol 2011;24:33-8.

32. Baldus $\mathrm{S}$, Heeschen $\mathrm{C}$, Meinertz $\mathrm{T}$, et al. Myeloperoxidase serum levels predict risk in patients with acute coronary syndromes. Circulation 2003;108:1440-5.

33. Zairis MN, Adamopoulou EN, Manousakis SJ, et al. The impact of hs C-reactive protein and other inflammatory biomarkers on long-term cardiovascular mortality in patients with acute coronary syndromes. Atherosclerosis 2007;194:397-402.

34. Varo N, de Lemos JA, Libby P, et al. Soluble CD40L: risk prediction after acute coronary syndromes. Circulation 2003;108:1049-52.

35. Block RC, Harris WS, Reid KJ, et al. EPA and DHA in blood cell membranes from acute coronary syndrome patients and controls. Atherosclerosis 2008;197:821-8.

36. Ballantyne C, Cushman M, Psaty B, et al. Collaborative meta-analysis of individual participant data from observational studies of Lp-PLA2 and cardiovascular diseases. Eur J Cardiovasc Prev Rehabil 2007;14:3-11.
37. Thompson A, Gao P, Orfei L, et al. Lipoprotein-associated phospholipase $\mathrm{A}(2)$ and risk of coronary disease, stroke, and mortality: collaborative analysis of 32 prospective studies. Lancet 2010;375:1536-44.

38. Cavusoglu E, Ruwende C, Chopra V, et al. Relationship of baseline plasma ADMA levels to cardiovascular outcomes at 2 years in men with acute coronary syndrome referred for coronary angiography. Coron Artery Dis 2009;20:112-17.

39. Biasucci LM, Vitelli A, Liuzzo G, et al. Elevated levels of interleukin-6 in unstable angina. Circulation 1996;94:874-7.

40. Schieffer B, Schieffer E, Hilfiker-Kleiner D, et al. Expression of angiotensin II and interleukin 6 in human coronary atherosclerotic plaques: potential implications for inflammation and plaque instability. Circulation 2000;101:1372-8.

41. Lindmark E, Diderholm E, Wallentin L, et al. Relationship between interleukin 6 and mortality in patients with unstable coronary artery disease: effects of an early invasive or noninvasive strategy. JAMA 2001;286:2107-13.

42. Nian $\mathrm{M}$, Lee $\mathrm{P}$, Khaper $\mathrm{N}$, et al. Inflammatory cytokines and postmyocardial infarction remodeling. Circ Res 2004;94:1543-53.

43. Ridker PM, Rifai N, Pfeffer M, et al. Elevation of tumor necrosis factor-alpha and increased risk of recurrent coronary events after myocardial infarction. Circulation 2000;101:2149-53.

44. Kunadian V, Ford GA, Bawamia B, et al. Vitamin D deficiency and coronary artery disease: a review of the evidence. Am Heart $J$ 2014;167:283-91.

45. Perez-Rivera JA, Pabon-Osuna P, Cieza-Borrella C, et al. Prognostic value of telomere length in acute coronary syndrome. Mech Ageing Dev 2012;133:695-7.

46. Calvert PA, Liew TV, Gorenne I, et al. Leukocyte telomere length is associated with high-risk plaques on virtual histology intravascular ultrasound and increased proinflammatory activity. Arterioscler Thromb Vasc Biol 2011;31:2157-64.

47. Berezikov E. Evolution of microRNA diversity and regulation in animals. Nat Rev Genet 2011;12:846-60.

48. Moldovan L, Batte KE, Trgovcich J, et al. Methodological challenges in utilizing miRNAs as circulating biomarkers. J Cell Mol Med 2014;18:371-90.

49. Valadi $\mathrm{H}$, Ekström $\mathrm{K}$, Bossios $\mathrm{A}$, et al. Exosome-mediated transfer of mRNAs and microRNAs is a novel mechanism of genetic exchange between cells. Nat Cell Biol 2007;9:654-9.

50. Vickers KC, Palmisano BT, Shoucri BM, et al. MicroRNAs are transported in plasma and delivered to recipient cells by high-density lipoproteins. Nat Cell Biol 2011;13:423-33.

51. Wagner J, Riwanto M, Besler C, et al. Characterization of levels and cellular transfer of circulating lipoprotein-bound microRNAs. Arterioscler Thromb Vasc Biol 2013;33:1392-400.

52. Turchinovich A, Burwinkel B. Distinct AGO1 and AGO2 associated miRNA profiles in human cells and blood plasma. RNA Biol 2012:9:1066-75.

53. D'Alessandra $\mathrm{Y}$, Devanna $\mathrm{P}$, Limana $\mathrm{F}$, et al. Circulating microRNAs are new and sensitive biomarkers of myocardial infarction. Eur Heart $J$ 2010;31:2765-73.

54. Wang GK, Zhu JQ, Zhang JT, et al. Circulating microRNA: a novel potential biomarker for early diagnosis of acute myocardial infarction in humans. Eur Heart J 2010;31:659-66.

55. De Rosa S, Fichtlscherer S, Lehmann R, et al. Transcoronary concentration gradients of circulating microRNAs. Circulation 2011;124:1936-44

56. Devaux $\mathrm{Y}$, Vausort M, McCann GP, et al. MicroRNA-150: a novel marker of left ventricular remodeling after acute myocardial infarction. Circ Cardiovasc Genet 2013;6:290-8.

57. Eitel I, Adams V, Dieterich P, et al. Relation of circulating MicroRNA-133a concentrations with myocardial damage and clinical prognosis in ST-elevation myocardial infarction. Am Heart $J$ 2012:164:706-14.

58. Widera C, Gupta SK, Lorenzen JM, et al. Diagnostic and prognostic impact of six circulating microRNAs in acute coronary syndrome. $J \mathrm{Mol}$ Cell Cardiol 2011:51:872-5.

59. Farb A, Burke AP, Tang AL, et al. Coronary plaque erosion without rupture into a lipid core. A frequent cause of coronary thrombosis in sudden coronary death. Circulation 1996;93:1354-63.

60. Burke AP, Farb A, Malcom GT, et al. Coronary risk factors and plaque morphology in men with coronary disease who died suddenly. N Engl J Med 1997;336:1276-82.

61. Nair A, Kuban BD, Tuzcu EM, et al. Coronary plaque classification with intravascular ultrasound radiofrequency data analysis. Circulation 2002;106:2200-6.

62. García-García HM, Mintz GS, Lerman A, et al. Tissue characterisation using intravascular radiofrequency data analysis: 
recommendations for acquisition, analysis, interpretation and reporting. Eurolntervention 2009;5:177-89.

63. Mintz GS, Kent KM, Pichard AD, et al. Contribution of inadequate arterial remodeling to the development of focal coronary artery stenoses. An intravascular ultrasound study. Circulation 1997;95:1791-8.

64. Huang D, Swanson EA, Lin CP, et al. Optical coherence tomography. Science 1991;254:1178-81.

65. Sinclair $\mathrm{H}$, Bourantas $\mathrm{C}$, Bagnall $\mathrm{A}$, et al. OCT for the identification of vulnerable plaque in acute coronary syndrome. JACC Cardiovasc Imaging 2015;8:198-209.

66. Prati F, Guagliumi G, Mintz GS, et al, Expert's OCT Review Document. Expert review document part 2: methodology, terminology and clinical applications of optical coherence tomography for the assessment of interventional procedures. Eur Heart J 2012;33:2513-20.

67. Tearney GJ, Regar E, Akasaka T, et al. Consensus standards for acquisition, measurement, and reporting of intravascular optical coherence tomography studies: a report from the International Working Group for Intravascular Optical Coherence Tomography Standardization and Validation. J Am Coll Cardiol 2012;59:1058-72.

68. Laurent S, Cockcroft J, Van Bortel L, et al. Expert consensus document on arterial stiffness: methodological issues and clinical applications. Eur Heart J 2006;27:2588-605.

69. Weber T, Auer J, O'Rourke MF, et al. Prolonged mechanical systole and increased arterial wave reflections in diastolic dysfunction. Heart 2006;92:1616-22.

70. Veerasamy M, Ford GA, Neely D, et al. Association of aging, arterial stiffness and cardiovascular disease: a review. Cardiol Rev 2014;22:223-32.

71. Lüscher TF, Barton M. Biology of the endothelium. Clin Cardiol 1997;20(Suppl 2):II-3-10.

72. Veerasamy M, Bagnall A, Neely D, et al. Endothelial dysfunction and coronary artery disease: a state of the art review. Cardiol Rev 2015;23:119-29.

73. Ross R. The pathogenesis of atherosclerosis: a perspective for the 1990s. Nature 1993;362:801-9.

74. Halcox JP, Schenke WH, Zalos G, et al. Prognostic value of coronary vascular endothelial dysfunction. Circulation 2002;106:653-8.

75. Schächinger V, Britten MB, Zeiher AM. Prognostic impact of coronary vasodilator dysfunction on adverse long-term outcome of coronary heart disease. Circulation 2000;101:1899-906.

76. Suwaidi JA, Hamasaki S, Higano ST, et al. Long-term follow-up of patients with mild coronary artery disease and endothelial dysfunction. Circulation 2000;101:948-54.

77. Kuvin JT, Patel AR, Sliney KA, et al. Assessment of peripheral vascular endothelial function with finger arterial pulse wave amplitude. Am Heart J 2003;146:168-74.

78. Lavie P, Schnall RP, Sheffy J, et al. Peripheral vasoconstriction during REM sleep detected by a new plethysmographic method. Nat Med 2000;6:606.

79. Nohria A, Gerhard-Herman M, Creager MA, et al. Role of nitric oxide in the regulation of digital pulse volume amplitude in humans. $J \mathrm{Appl}$ Physiol 2006;101:545-8.
80. Noon JP, Haynes WG, Webb DJ, et al. Local inhibition of nitric oxide generation in man reduces blood flow in finger pulp but not in hand dorsum skin. J Physiol 1996;490(Pt 2):501-8.

81. Rubinshtein R, Kuvin JT, Soffler M, et al. Assessment of endothelia function by non-invasive peripheral arterial tonometry predicts late cardiovascular adverse events. Eur Heart J 2010;31:1142-8.

82. Lorenz MW, Markus HS, Bots ML et al. Prediction of clinical cardiovascular events with carotid intima-media thickness: a systematic review and meta-analysis. Circulation 2007;115: 459-67.

83. Carpenter M, Sinclair H, Kunadian V. Carotid intimal media thickness and its utility as a predictor of cardiovascular disease: a review of evidence. Cardiol Rev 2016;24:70-5.

84. Kalay N, Yarlioglues M, Ardic I, et al. The assessment of atherosclerosis on vascular structures in patients with acute coronary syndrome. Clin Invest Med 2010;33:E36-43.

85. Den Ruijter HM, Peters SA, Anderson TJ, et al. Common carotid intima-media thickness measurements in cardiovascular risk prediction: a meta-analysis. JAMA 2012;308:796-803.

86. Simons PC, Algra A, Bots ML, et al. Common carotid intima-media thickness and arterial stiffness: indicators of cardiovascular risk in high-risk patients. The SMART Study (Second Manifestations of ARTerial disease). Circulation 1999;100:951-7.

87. Zhang $\mathrm{Y}$, Safar ME, laria $\mathrm{P}$, et al. Prevalence and prognosis of left ventricular diastolic dysfunction in the elderly: the PROTEGER study. Am Heart J 2010;160:471-8.

88. Wharton $\mathrm{G}$, Steeds R, Allen J, et al. A minimum dataset for a standard adult transthoracic echocardiogram: a guideline protocol from the British Society of Echocardiography. Echo Res Pract 2015;2:G9-24.

89. Thygesen K, Alpert JS, White HD, et al. Universal definition of myocardial infarction. Eur Heart J 2007;28:2525-38.

90. Mehran R, Rao SV, Bhatt DL, et al. Standardized bleeding definitions for cardiovascular clinical trials: a consensus report from the Bleeding Academic Research Consortium. Circulation 2011;123:2736-47.

91. Hsieh FY, Lavori PW. Sample-size calculations for the Cox proportional hazards regression model with nonbinary covariates. Control Clin Trials 2000;21:552-60.

92. British Cardiovascular Intervention Society. BCIS audit return 2011. London (UK): British Cardiovascular Intervention Society, 2011.

93. Harrell FE Jr, Lee KL, Pollock BG. Regression models in clinical studies: determining relationships between predictors and response. J Natl Cancer Inst 1988;80:1198-202.

94. Cook NR, Ridker PM. Advances in measuring the effect of individual predictors of cardiovascular risk: the role of reclassification measures. Ann Intern Med 2009;150:795-802.

95. Picard RR, Cook RD. Cross-validation of regression-models. J Am Stat Assoc 1984;79:575-83.

96. lasonos A, Schrag D, Raj GV, et al. How to build and interpret a nomogram for cancer prognosis. J Clin Oncol 2008;26:1364-70.

97. World Medical Association. World Medical Association Declaration of Helsinki: ethical principles for medical research involving human subjects. JAMA 2013;310:2191-4. 